\title{
THE GROUP OF HOMOMORPHISMS OF ABELIAN TORSION GROUPS
}

\author{
M.W. LEGG \\ Department of Mathematics \\ Moorhead State University \\ Moorhead, Minnesota 56560 \\ U.S.A. \\ (Received February 10, 1978)
}

ABSTRACT. Let $G$ and $A$ be abelian torsion groups. In [5], R. S. Pierce develops a complete set of invariants for $\operatorname{Hom}(G, A)$. To compute these invariants he introduces, and uses extensively, the group of small homomorphisms of G into A. Aloo, using some of Pierce's methods, Fuchs characterizes this group in [1]. Our purpose in this paper is to characterize $\operatorname{Hom}(G, A)$ in what seems to be a more natural manner than either of the treatments just mentioned.

KEY WORDS AND PHRASES. Abelian Torsion Groups, Group of Homomorphisms, and Ulm invariants. AMS (MOS) SUBJECT CLASSIFICATION (1970) CODES. 20K10, $20 K 30$.

1. INTRODUCTION.

It is well-known (Harrision [2]) that $\operatorname{Hom}(\mathrm{G}, \mathrm{A})$ is an algebraically compact group. In [3] Legg and Walker use elementary techniques to show that algebraically compact groups are characterized by their U1m invariants and their torsion free 
numbers. In this paper we will make use of methods introduced in [3] to calculate these invariants for $\operatorname{Hom}(G, A)$.

The notation used here will mainly conform to that of Fuchs [1]. In this paper group means additive abelian group. The maximal divisible subgroup of the group $G$ will be denoted $d G$. If $d G=0, G$ is called reduced. We will use $G$ to denote the p-primary component of $G$ and $t G$ to represent the torsion subgroup of $G$. If $Q$ is the group of rationals and $Z$ is the group of integers, then $(Q / Z)_{p}$ is denoted $Z\left(p^{\infty}\right)$. $Z(n)$ denotes the cyclic group of order $n$.

\section{PRELIMINARIES.}

A group $G$ is called algebraically compact (Maranda [4]) if for every group $X$, any homomorphism from a pure subgroup of $\mathrm{X}$ to $\mathrm{G}$ can be extended to a homomorphism of $\mathrm{X}$ to $\mathrm{G}$.

We now recall two definitions and the main result from the paper by Legg and Walker that was mentioned above.

Let $G$ be a group, $p$ a prime, and $n$ a non-negative integer. Let $G_{p}(n)$ denote the dimension of $\left(p^{n} G\right)[P] /\left(p^{n+1} G\right)[P]$ as a vector space over the integers modulo $p$. That is, $G_{p}(n)$ is the $n$-th U1m invariant of $G$ (with respect to $p$ ).

If $G$ is a group and $p$ is a prime, then $G_{0}(p)$ shall denote the dimension of $G /(p G+t G)$ as a vector space over the integers modulo $p$. We call $G_{0}(p)$ the $p-t h$ torsion free number of $G$.

The Ulm invariants and the torsion free numbers are a complete set of invariants for algebraically compact, groups. That is, two algebraically compact groups $A$ and $B$ are isomorphic if and only if

$$
\begin{aligned}
& \text { (i) } d A \cong d B \\
& \text { (ii) } A_{0}(p)=B_{0}(p) \text { for all primes } p \text {, and } \\
& \text { (iii) } A_{p}(n)=B_{p}(n) \text { for all primes } p \text { and all non-negative } \\
& \text { integers } n .
\end{aligned}
$$


We now proceed to calculate $\operatorname{Hom}_{p}(G, A)(n)$ and $\operatorname{Hom}_{0}(G, A)(p)$ for the algebrically compact $\operatorname{Hom}(G, A)$.

It will suffice, as we shall show later, to confine our calculations to pprimary groups. So, for the time being, all groups will be understood to be of this type.

Our calculation of the Ulm invariants of $\operatorname{Hom}(G, A)$ requires that we determine the adjusted component of $\operatorname{Hom}(G, A)$. This result is interesting in itself.

\section{THE ADJUSTED COMPONENT OF HOM $(G, A)$.}

The following definition and results are due to Harrison [2]. These results were subsequently obtained by Legg and Walker [3] using quite elementary methods. A reduced algebraically compact group is called adjusted if it has no torsion free direct summands. A reduced algebraically compact group $A$ is the direct sum of an adjusted algebraically compact group and a torsion free algebraically compact group. The adjusted component of $A$ is unique. Further, $G$ is the adjusted part of $A$ if and only if $\mathrm{G} / \mathrm{tA}=\mathrm{d}(\mathrm{A} / \mathrm{tA})$. (For an arbitrary $\mathrm{G}$, the adjusted component of the reduced part of $G$ is called the adjusted component of $G$ ).

The adjusted component of $\operatorname{Hom}(G, A)$ will be denoted $\operatorname{Adj}(G, A)$ and its elements will be called adjusted homomorphisms.

THEOREM 3.1. $\operatorname{Adj}(G, A)$ is the set of all $\alpha \varepsilon \operatorname{Hom}(G, A)$ such that for each integer $m \geq 0$ there is an integer $n \geq 0$ such that (i) $\alpha\left(p^{n} G\right) \subseteq p^{m+n} A$ and (ii) $\left(p^{n} G\right)\left[p^{m}\right] \subseteq \operatorname{Ker} \alpha$.

PROOF. Let $\mathbf{S}(\mathrm{G}, \mathrm{A})$ be the subgrbup of Hom $(G, A)$ consisting of those homomorphisms that satisfy conditions (i) and (ii) of the theorem. Let $\alpha \varepsilon \operatorname{Adj}(G, A)$ and $m \geq 0$ be an integer.

Since $\operatorname{Adj}(G, A) / t \operatorname{Hom}(G, A)=d(\operatorname{Hom}(G, A) / t \operatorname{Hom}(G, A))$, there is a $B \varepsilon \operatorname{Adj}(G, A)$ and an integer $n \geq 0$ so that $p^{n}\left(\alpha-p^{m} B\right)=0$. It follows that $\operatorname{Adj}(G, A) \subseteq S(G, A)$.

By the remarks preceding this theorem, we will be done if we show that 
$\mathrm{S}(\mathrm{G}, \mathrm{A}) / \mathrm{tHom}(\mathrm{G}, \mathrm{A})$ is divisible.

Since $\operatorname{Hom}(G, A)$ is $q$-divisible for primes $q \neq p$ and since $\operatorname{Hom}(G, A) / S(G, A)$ is clearly q-torsion free, it follows that $S(G, A) / t \operatorname{Hom}(G, A)$ is q-divisible. We show now that it is $p$-divisible. Let $\alpha \in S(G, A)$ and choose $n \geq 0$ such that $\alpha\left(p^{n} G\right) \subseteq p^{n+1} A$ and $\left(p^{n} G\right)[p] \subseteq \operatorname{Ker} \alpha$.

Let $B=\sum_{i=1}^{\infty} B_{i}$ and $C=\sum_{i=1}^{\infty} C_{i}$ be basic in $G$ and $A$ respectively, where $B_{i}$ and $C_{i}$ are direct sums of cyclic groups each of order $p^{1}$. Then we can write $G=B_{1} \oplus \ldots \oplus B_{n+1} \oplus G_{n+1}$ and $A=c_{1} \oplus \ldots \oplus c_{n+1} \oplus A_{n+1}$. Since $\alpha\left(p^{n} G\right) \subseteq p^{n+1} A=p^{n+1} A_{n+1}$, we can define $\beta_{1}: p^{n+1} G_{n+1}+A_{n+1}$ by $\beta_{1}\left(p^{n+1} x\right)=\alpha\left(p^{n} x\right)$. It is easy to check that $\beta_{1}$ is welldefined. Let $D$ be the divisible hull of $A_{n+1}$. Since $p^{n+1} G_{n+1}$ is essential in $G_{n+1}$, we can extend $B_{1}$ to a homomorphism $\beta_{2}$ from $G_{n+1}$ to $D$. Also $p^{n+1} A_{n+1}$ is essential in $A_{n+1}$ so that $B_{2}\left(G_{n+1}\right) \subseteq A_{n+1}$.

Finally, define the homomorphism $\beta: G \rightarrow A$ by $\beta(x)=0$ for $x \in B_{1} \oplus \ldots \oplus B_{n+1}$ and $\beta(x)=\beta_{2}(x)$ for $x \in G_{n+1}$. Then $p^{n}(\alpha-p \beta)=0$, since $\beta\left(p^{n+1} x\right)=\alpha\left(p^{n} x\right)$ for $\mathbf{X} \varepsilon \mathrm{G}$.

It remains to show that $\beta \in S(G, A)$. It is routine that $\mathrm{p}_{\alpha}^{\mathrm{n}} \in \mathrm{S}(\mathrm{G}, \mathrm{A})$; thus $\mathrm{p}^{\mathrm{n}+1_{B}} \in \mathrm{S}(G, A)$. We must show that $\operatorname{Hom}(G, A) / S(G, A)$ is $p$-torsion free. Suppose $\gamma \varepsilon \operatorname{Hom}(\mathrm{G}, \mathrm{A})$ and $\mathrm{P} \gamma \varepsilon \mathrm{S}(\mathrm{G}, \mathrm{A})$. Let $\mathrm{m} \geq 0$ be an integer. Choose $\mathrm{n} \geq 0$ such that pr $\left(p^{n} G\right) \subseteq p^{m+n+1} A$ and $\left(p^{n} G\right)\left[p^{m+1}\right] \subseteq$ Ker pr. Then $\gamma\left(p^{n+1} G\right)=\operatorname{pr}\left(p^{n} G\right) \subseteq p^{m+n+1} A \subseteq p^{m+n} A$. Also $\left(p^{n+1} G\right)\left[p^{m}\right] \subseteq \operatorname{Ker} \gamma$, since $p^{n+1} x \in\left(p^{n+1} G\right)\left[p^{m}\right]$ implies that $\gamma\left(p^{n+1} x\right)=p \gamma\left(p^{n} x\right)=0$. Thus $\gamma \varepsilon s(G, A)$, as we wished to show. Hence $p^{n+1} B \in S(G, A)$ implies that $S(G, A) / t \operatorname{Hom}(G, A)$ is divisible. The proof is complete.

We next investigate the functorial behavior of $\operatorname{Adj}(G, A)$. The proof of our first lemma depends on the following result [3]: If $A$ is pure in $B$, then there 
exists a group $C$, containing $B$, with $C / A$ divisible and $A$ pure in $C$.

LEMMA 3.2. If $A$ is pure in $B$ and $\phi \varepsilon \operatorname{Adj}(A, X)$, then $\phi$ can be extended to an adjusted homomorphism of $B$ into $X$.

PROOF. It is immediate that the restriction of an adjusted homomorphism is adjusted. Hence, by the remark preceding this lemma, we can assume that B/A is divisible.

Let $b \in B$ with $o(b)=p^{m}$. Choose $n$ so that $\left(p^{n} A\right)\left[p^{m}\right] \subseteq \operatorname{Ker} \phi$. Since A is pure in $B$ and $B / A$ is divisible, there exist $b_{n} \varepsilon B$ and $a_{n} \varepsilon A$ such that $b=p^{n} b_{n}+a_{n}$ and $o\left(b_{n}\right)=o\left(b_{n}+A\right)$. Note that $b+A=p^{n} b_{n}+A$. Thus, since $o\left(b_{n}\right)=o\left(b_{n}+A\right)$, the fact that $p^{m}=0$ implies that $p^{m+n_{b}}=0$. Hence $a_{n} \varepsilon A\left[p^{m}\right]$. Now define $\psi: B \rightarrow X$ by $\psi(b)=\phi\left(a_{n}\right)$.

Suppose also $\left(p^{k} A\right)\left[p^{m}\right] \subseteq \operatorname{Ker} \phi$, and assume $k \geq n$. Write $b=p^{k_{k}}+a_{k}$, with $o\left(b_{k}\right)=o\left(b_{k}+A\right)$. Then $a_{k} \varepsilon A\left[p^{m}\right]$. We have that $p^{n}\left(b_{n}-p^{k-n_{b}}\right)=a_{k}-a_{n} \cdot$ since $A$ is pure in $B$, there is an a $\varepsilon A$ so that $p^{n} a=a_{k}-a_{n}$. Thus $a_{k}-a_{n} \varepsilon\left(p^{n} A\right)\left[p^{m}\right]$. Hence $\phi\left(a_{n}\right)=\phi\left(a_{k}\right)$ so that $\psi$ is well-defined. It is routine to check that $\psi$ is a homomorphism.

Finally, we show that $\psi$ is adjusted. Let $m \geq 0$ be an integer. Then there is an integer $n \geq 0$ so that $\left(p^{n} A\right)\left[p^{m}\right] \subseteq \operatorname{Ker} \phi$ and $\phi\left(p^{n} A\right) \subseteq p^{m+n} x$. Let $b \varepsilon\left(p^{n} B\right)\left[p^{m}\right]$ with o (b) $=p^{k}$ and $b=p^{n} b_{1}$. Choose $b_{n} \varepsilon B, a_{n} \varepsilon A$ so that $b=p^{n} b_{n}+a_{n}$ and $o\left(b_{n}\right)=o\left(b_{n}+A\right)$. Note that $a_{n} \varepsilon A\left[p^{k}\right] \subseteq A\left[p^{m}\right]$. We have $p^{n}\left(b_{1}-b_{n}\right)=a_{n}$ so that, by purity, there is an a $\varepsilon$ A such that $p^{n} a=a_{n}$. It follows that $a_{n} \varepsilon\left(p^{n} A\right)\left[p^{m}\right]$. Hence $\psi(b)=\phi\left(a_{n}\right)=0 ; 1 . e$. we have shown that $\left(p^{n} B\right)\left[p^{m}\right] \subseteq \operatorname{Ker} \psi$.

Now let $b \in p^{n_{B}}$, say $b=p^{n_{1}}$, with $o(b)=p^{k}$. Choose $s>n$ so that $\left(p^{s} A\right)\left[p^{k}\right] \subseteq \operatorname{Ker} \phi . \quad$ Let $b=p^{s_{b}}+a_{s}$ with o(b $)=o\left(b_{s}+A\right)$. Then $p^{n_{1}} b_{1}-p^{s_{b}} b_{s}=a_{s}$ so that, by purity, there is an a $\varepsilon A$ such that $p^{n} a=a_{s}, 1 . e . a_{s} \varepsilon p^{n} A$. It follows that $\psi(b)=\phi\left(a_{s}\right) \varepsilon p^{m+n} x$. Thus $\psi\left(p^{n} B\right) \subseteq p^{m+n} x$.

We have shown that $\psi \varepsilon \operatorname{Adj}(B, X)$. Since $\psi$ extends $\phi$, the lemma is proved. 
If $\phi \varepsilon \operatorname{Adj}(X, Y)$ and $\alpha \varepsilon \operatorname{Hom}(A, X)$, then it is routine to show that $\phi \alpha \varepsilon \operatorname{Adj}(A, Y)$. Thus $\alpha$ determines a homomorphism $\alpha^{*}: \operatorname{Adj}(X, Y) \rightarrow \operatorname{Adj}(A, Y)$, where $\alpha *(\phi)=\phi \alpha$.

THEOREM 3.3. Let $0 \stackrel{2 *}{\rightarrow} A \rightarrow B \stackrel{n}{\rightarrow} B / A \rightarrow 0$ be pure exact. Then (*) $0 \rightarrow \operatorname{Adj}(B / A, X) \eta^{*} \operatorname{Adj}(B, X) l^{*} \operatorname{Adj}(A, X) \rightarrow 0$ is split exact for arbitrary $X$

PROOF. Clearly $\eta^{*}$ is a monomorphism and $\operatorname{Im} \eta^{*} \subseteq \operatorname{Ker} \imath^{*}$. Let $\phi \varepsilon \operatorname{Ker} \imath^{*}$ Then $A \subseteq \operatorname{Ker} \phi$. Hence $\phi=\hat{\phi} n$, where $\hat{\phi}: B / A \rightarrow X: b+A \rightarrow \phi(b)$.

We must show that $\hat{\phi}$ is adjusted. Let $m \geq 0$ be an integer. Since $\phi$ is adjusted, there is an integer $n \geq 0$ such that $\left(p^{n} B\right)\left[p^{m}\right] \subseteq \operatorname{Ker} \phi$ and $\phi\left(p^{n} B \subseteq p^{m+n} x\right.$. Let $b+A \varepsilon\left(p^{n}(B / A)\right)\left[p^{m}\right]$, say $b+A=p^{n} b_{1}+A$ with $o\left(b_{1}\right)=o\left(b_{1}+A\right)$. Then $p^{n} b_{1} \varepsilon\left(p^{n} B\right)\left[p^{m}\right]$ so that $\phi(b+A)=\phi\left(p^{n} b_{1}\right)=0$. Hence $\left(p^{n}(B / A)\right)\left[p^{m}\right] \subseteq \operatorname{Ker} \phi$. Clearly $\phi\left(p^{n}(B / A)\right) \subseteq p^{m+n} x$. Thus $\phi$ is adjusted. Hence $\phi$ is adjusted, which shows that $\operatorname{Ker} \imath * \subseteq \operatorname{Im} \eta^{*}$. We have shown exactness at $\operatorname{Adj}(B, X)$.

The fact that $\imath *$ is onto is a consequence of Lemma 3.2 .

By Fuchs $[1], \eta^{*}(\operatorname{Hom}(B / A, X))$ is pure in $\operatorname{Hom}(B, X)$. Since $\operatorname{Adj}(B / A, X)$ is a summand of $\operatorname{Hom}(B / A, X)$ and $\eta *(\operatorname{Adj}(B / A, X)) \subseteq \operatorname{Adj}(B, X)$, it follows that $\eta *(\operatorname{Adj}(B / A, X))$ is pure in $\operatorname{Adj}(B, X)$.

Hence (*) is pure exact. Since $\operatorname{Adj}(B / A, X)$ is algebraically compact, (*) is split exact. The theorem is proved.

Two important results follow immediately from Theorem 3.3.

THEOREM 3.4. Let $B$ be basic in $G$. Then, for $\operatorname{arbitrary} A, \operatorname{Adj}(G, A) \cong \operatorname{Adj}(B, A)$.

PROOF. Since $0 \rightarrow \mathrm{B} \rightarrow \mathrm{G} \rightarrow \mathrm{G} / \mathrm{B} \rightarrow 0$ is pure exact, Theorem $3.3 \mathrm{y} 1 \mathrm{elds}$ $\operatorname{Adj}(G, A) \cong \operatorname{Adj}(B, A) \bigoplus \operatorname{Adj}(G / B, A)$. But $\operatorname{Adj}(G, B / A)=0$, since Hom $(G / B, A)$ is torsion free.

COROLLARY 3.5. If $B$ is basic in $G$, then $\operatorname{Hom}(G, A){ }_{p}(n)=\operatorname{Hom}(B, A)_{p}(n)$ for all Integers $n \geq 0$.

PROOF. This follows from Theorem 3.4 and the observation that the Ulm invariants 
of an algebraically compact group are the same as the U1m invariants of its adjusted part.

We are now ready to calculate the Ulm invariants for $\operatorname{Hom}(G, A)$.

\section{THE ULM INVARIANTS OF HOM $(G, A)$.}

Let $G$ and $A$ be p-groups with $B$ basic in $G$. Let $B=\sum_{1=1}^{\infty} B_{1}$ where $\begin{aligned} B_{i}=\sum_{p}^{\sum}(i-1) & Z\left(p^{i}\right) . \text { Then } \\ & \operatorname{Hom}(B, A) \cong \prod_{i=1}^{\infty} \operatorname{Hom}\left(B_{i}, A\right) \cong \prod_{i=1}^{\infty} G_{p}(i-1) \operatorname{Hom}\left(Z\left(p^{i}\right), A\right) \cong \prod_{1}^{\infty} G_{p} \prod_{1-1)} A\left[p^{i}\right] .\end{aligned}$ It follows that

$$
\begin{aligned}
& \operatorname{Hom}(G, A)_{p}(n)=\operatorname{Hom}(B, A)_{p}(n) \\
= & \operatorname{dim} \prod_{i=1}^{\infty} G_{p}(i-1)\left(p^{n}\left(A\left[p^{i}\right]\right)\right)[p] /\left(p^{n+1}\left(A\left[p^{i}\right]\right)\right)[p] \\
= & \operatorname{dim} \prod_{i=1}^{\infty} G_{p}(i-1) A\left[p^{i}\right]_{p}(n) Z(p) .
\end{aligned}
$$

Observe that for $m>n,\left(p^{n}\left(A\left[p^{m}\right]\right)\right)[p]=\left(p^{n} A\right)[p]$ and for $m \leq n, p^{n}\left(A\left[p^{m}\right]\right)=0$. Thus

$$
A\left[p^{i}\right]_{p}(n)=\left\{\begin{array}{ll}
0 & \text { if } i \leq n \\
r\left(p^{n} A\right) & \text { if } i=n+1 \\
A_{p}(n) & \text { if } i>n+1
\end{array}\right\} \text {, where }
$$

$r\left(p^{n} A\right)$ denotes the rank of $p^{n} A$. Hence we have proved the following theorem.

THEOREM 4.1. If $G$ and $A$ are $p$-groups, then $\operatorname{Hom}(G, A)_{p}(n)=$

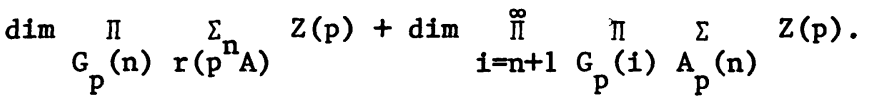

In Theorem 4.1, as in certain subsequent theorems, we prefer not to compute the dimensions of the $Z(p)$-spaces involved; such computations are easy, but the resulting expressions are clumsy.

5. THE TORSION FREE NUMBER OF HOM $(G, A)$.

The computation of $\operatorname{Hom}(G, A)_{0}(p)$ will be done in three steps by the following 
lemmas.

LEMMA 5.1. Let $G$ and $A$ be divisible. Then $\operatorname{Hom}(G, A)_{0}(p)=\operatorname{dim} \prod_{r(G)} \underset{r(A)}{\Sigma} Z(p)$.

PROOF. Note that $\operatorname{Hom}(G, A) \cong \operatorname{Hom}\left(\Sigma \quad \mathrm{Z}\left(\mathrm{p}^{\infty}\right) ; \Sigma \quad \mathrm{Z}\left(\mathrm{p}^{\infty}\right)\right)$

$\cong \underset{r(G)}{\pi} \operatorname{Hom}\left(Z\left(\mathrm{p}^{\infty}\right), \underset{\mathrm{r}(\mathrm{A})}{\sum} \mathrm{Z}\left(\mathrm{p}^{\infty}\right)\right)$. Since $\operatorname{dim} \operatorname{Hom}\left(\mathrm{Z}\left(\mathrm{p}^{\infty}\right), \underset{\mathrm{r}(\mathrm{A})}{\sum} \mathrm{Z}\left(\mathrm{p}^{\infty}\right)\right)=\mathrm{r}(\mathrm{A})$

(cf. [2] or [3]), the result follows.

The final rank of a $p$-group $G$ is defined to be $\operatorname{fr}(G)=\min \left\{r\left(p^{n} G\right) \mid n=0,1,2, \ldots\right\}$ (cf. Fuchs [1]).

LEMMA 5.2. Let $G$ be reduced and $A$ be divisible. Then $\operatorname{Hom}(G, A)_{0}(p)=\operatorname{dim} \underset{\operatorname{fr}(G)}{\Pi} \underset{r(A)}{\sum} Z(p)$.

PROOF. If $\mathrm{fr}(G)$ is finite, it is zero and the result is clear. Hence we can assume that $\operatorname{fr}(G)$ is infinite. By Fuchs $[1]$ we can write $G=G_{1} \oplus G_{2}$, where $G_{1}$ is bounded and $r\left(G_{2}\right)=\operatorname{fr}\left(G_{2}\right)=\operatorname{fr}(G)$. Hence, since $\operatorname{Hom}(G, A)_{0}(p)=\operatorname{Hom}(G, A)_{0}(p)$, we can assume that $\operatorname{fr}(G)=r(G)$. Let $B$ be basic in $G$ such that $r(G / B)=r(G)$. Then $0 \rightarrow B \rightarrow G \rightarrow G / B \rightarrow 0$ pure exact and A divisible implies that $0 \rightarrow \operatorname{Hom}(G / B, A) \rightarrow \operatorname{Hom}(G, A) \rightarrow \operatorname{Hom}(B, A) \rightarrow 0$ is split exact. Thus $\operatorname{Hom}(G, A)_{0}(p)=\operatorname{Hom}(G / B, A)_{0}(p)+\operatorname{Hom}(B, A)_{0}(p)$. Since $r(B) \leq r(G)=r(G / B)$, there is a monomorphism from $B$ to $G / B$, hence an epimorphism of $\operatorname{Hom}(G / B, A)$ onto $\operatorname{Hom}(B, A)$. Since $\operatorname{Hom}(G / B, A)$ is torsion free, it follows that $\operatorname{Hom}(G / B, A)_{0}(p) \geq \operatorname{Hom}(B, A)_{0}(p)$. Thus, since $\operatorname{Hom}(G / B, A)_{0}(p)$ is infinite, we have $\operatorname{Hom}(G, A)_{0}(p)=\operatorname{Hom}(G / B, A)_{0}(p)$. This yields $\operatorname{Hom}(G, A)_{0}(p)=\operatorname{dim} \underset{\operatorname{fr}(G)}{\pi} \underset{r(A)}{\sum} Z(p)$ on application of Lemma 5.1.

The final rank of a basic subgroup of a p-group $G$ is an invariant of $G$ and is called the critical number of G. We shall denote it $\operatorname{cr}(G)$ (cf. Szele [6]).

LEMMA 5.3. Let $G$ and $A$ be reduced. Then $\operatorname{Hom}(G, A)_{0}(p)=\operatorname{dim} \prod_{\operatorname{cr}(G)} \sum_{\operatorname{cr}(A)} Z(p)$. PROOF. Let $B$ be basic in $G$ and $C$ basic in $A$. Let $\operatorname{cr}(G)=\alpha$ and $\operatorname{cr}(A)=\beta$. 
If $\alpha$ is finite it is zero and $B$ is bounded. Thus $G$ is bounded; hence so is Hom(G,A). It follows that $\operatorname{Hom}(G, A)_{0}(p)=0$. Similarily, if $B$ is finite the result follows. Hence we may take both $\alpha$ and $\beta$ to be infinite. By remarks similar to those at the beginning of the previous proof, we can assume that $r(B)=f r(B)$ and $r(C)=f r(C)$. $\operatorname{Clearly} \operatorname{Hom}(G, A)_{0}(P) \leq|\operatorname{Hom}(G, A)| \leq|\operatorname{Hom}(B, A)|$. Now $|B|=\alpha,|C|=B$, and $|A| \leq|C|^{H_{0}}=\beta^{H_{0}}$ (Fuchs [1]). Thus $|\operatorname{Hom}(B, A)| \leq\left(\beta^{K_{0}}\right)^{\alpha}=\beta^{\alpha}$, so that

$\operatorname{Hom}(G, A)_{0}(p) \leq \beta^{\alpha}$. We will reverse this inequality by constructing $\beta^{\alpha}$ elements in $\operatorname{Hom}(G, A) /(p H o m(G, A)+t \operatorname{tom}(G, A))$.

Let $B=\sum_{i=1}^{\infty} B_{i}$ and $C=\sum_{i=1}^{\infty} C_{i}$ in the usual notation.

Let $\left\{b_{i_{j}} \mid j \varepsilon J_{i}\right\}$ and $\left\{c_{i_{j}} \mid j \varepsilon K_{i}\right\}$ be bases for $B_{i}$ and $c_{i}$ respectively. We note that, for each $n, \sum_{i=n}^{\infty}\left|J_{i}\right| \geq \alpha$ and $\sum_{i=n}^{\infty}\left|k_{i}\right| \geq \beta$. It will be clear from the proof that we can assume that $\left|k_{i}\right|>1$ for each $i$. Choose a subsequence $\left\{k_{i}\right\}_{i=1}^{\infty}$ of $\left\{k_{i}\right\}_{i=1}^{\infty}$ such that $\left\{\left|k_{i}\right|\right\}_{j=1}^{\infty}$ is non-decreasing and $\sum_{j=1}^{\infty}\left|k_{i j}\right|=\beta ; \operatorname{denote}\left|k_{i_{j}}\right|$ by $\beta_{j}$. Next choose a subsequence $\left\{J_{i}\right\}_{j=1}^{\infty}$ of $\left\{J_{i}\right\}_{i=1}^{\infty}$ such that $\left\{\left|J_{i j}\right|\right\}_{j=1}^{\infty}$ is non-decreasing, such that $\sum_{j=1}^{\infty}\left|J_{i j}\right|=\alpha$, and so that the common order of the generators indexed by $J_{i_{j}}$ is greater than the order of the generators indexed by $k_{i_{j}} ; \operatorname{let}\left|J_{i_{j}}\right|=\alpha_{j}$. Let $\mathrm{J}_{i_{j}}$ index $\mathrm{B}_{i_{j}}$ and $\mathrm{K}_{i_{j}}$ index $\mathrm{C}_{i_{j}}$. Let $\delta_{j}: \mathrm{B}_{i_{j}} \rightarrow \mathrm{C}_{i_{j}}$ be a homomorphism induced by a map from the generators of $B_{i}$ to the generators of $C_{i_{j}}$. Each sequence $\left\{\delta_{j}\right\}_{j=1}^{\infty}$ induces a homomorphism, say $\delta$, from $B$ to $C$, namely homomorphism that is $\delta_{j}$ on $B_{i}$ and zero on the other components $B_{i}$ of $B$. Call two such homomorphisms $\delta=\left\{\delta_{j}\right\}_{j=1}^{\infty}$ and $\delta^{\prime}=\left\{\delta_{j}^{?}\right\}_{j=1}^{\infty}$ equivalent if $\delta_{j}=\delta_{j}^{\prime}$ for almost all $j$.

It follows that the cardinality of the set of equivalence classes is $\prod_{j=1}^{\infty} B_{j}^{\alpha}$ and it is routine to show that $\prod_{j=1}^{\infty} B_{j}^{\alpha}=\beta^{\alpha}$. 
Now if $\phi$ is an epimorphism from $G$ to $B$ and $\delta, \delta^{\prime}$ are two inequivalent maps, then $\delta \phi \neq \delta^{\prime} \phi$. Suppose $\delta \phi-\delta^{\prime} \phi=p \gamma+\tau$, where $\gamma, \tau \varepsilon$ Hom $(G, A)$ and $p^{n} \tau=0$. Then $\mathrm{p}^{\mathrm{n}}\left(\delta \phi-\delta^{\prime} \phi\right)=\mathrm{p}^{\mathrm{n}+1} \gamma$. According to the construction, there is a generator, say $c_{i_{k}}$ of order greater than $\mathrm{p}^{\mathrm{n}}$ as a component of some element in the image of $\delta-\delta^{\prime}$.

Thus there is an $x \in G$ such that $c_{i_{k}}$ is a component of $\left(\delta \phi-\delta^{\prime} \phi\right)(x)$. Since $c_{i_{k}}$ has height zero, $p^{n}\left(\delta \phi-\delta^{\prime} \phi\right)(x)$ has height exactly n. But $p^{n}\left(\delta \phi-\delta^{\prime} \phi\right)(x)=p^{n+1} \gamma(x)$ which gives a contradiction. Hence no such $\gamma$ and $\tau$ exist. We have shown that if $\delta$ and $\delta^{\prime}$ are inequivalent maps from $B$ to $C$, then $\delta \phi$ and $\delta^{\prime} \phi$ determine different cosets in $\operatorname{Hom}(G, A) /(p H o m(G, A)+t \operatorname{Hom}(G, A))$. Thus $B^{\alpha} \leq \mid \operatorname{Hom}(G, A) /(p H o m(G, A)+$ $\operatorname{tHom}(G, A)) \mid=\operatorname{Hom}(G, A)_{0}(p)$. The result follows.

THEOREM 5.4. If $G$ and $A$ are $p$-groups, then

$$
\operatorname{Hom}(G, A)_{0}(p)=\operatorname{dim} \prod_{\operatorname{cr}(G)} \underset{\operatorname{cr}(A)}{\sum} Z(p)+\operatorname{dim} \prod_{\operatorname{fr}(G)} \underset{r(d A)}{\sum} Z(p) .
$$

PROOF. Let $G=G_{1} \oplus \mathrm{dG}$ and $A=A_{1} \oplus \mathrm{dA}$. Then

$$
\operatorname{Hom}(G, A) \cong \operatorname{Hom}\left(G_{1}, A_{1}\right) \oplus \operatorname{Hom}\left(G_{1}, d A\right) \oplus \operatorname{Hom}(d G, d A) .
$$

Fom the preceding three lemmas we obtain

$$
\operatorname{Hom}(G, A)_{0}(p)=\operatorname{dim} \prod_{\operatorname{cr}\left(G_{1}\right)} \sum_{\operatorname{cr}\left(A_{1}\right)} Z(p)+\operatorname{dim} \prod_{\operatorname{fr}\left(G_{1}\right)} \sum_{r(d A)} Z(p)+\operatorname{dim} \underset{r(d G)}{\pi} \underset{r(d A)}{\Sigma} Z(p) .
$$

Using the relations $\operatorname{cr}(G)=\operatorname{cr}\left(G_{1}\right), \operatorname{cr}(A)=\operatorname{cr}\left(A_{1}\right), \operatorname{fr}(G)=f r\left(G_{1}\right)+r(d G)$, the result follows.

Since $\operatorname{Hom}(G, A)$ is $q$-divisible for all primes $q \neq p, \operatorname{Hom}(G, A)_{0}(p)$ and each $\operatorname{Hom}(G, A)_{q}(n)$ are zero for such $q$. Thus we can summarize our results in the following theroem.

THEOREM 5.5. If $G$ and $A$ are $p$-groups, then $\operatorname{Hom}(G, A)$ is algebraically compact. Complete invariants for $\operatorname{Hom}(G, A)$ are

$$
\operatorname{Hom}(G, A)_{0}(p)=\operatorname{dim} \prod_{\operatorname{cr}(G)} \underset{\operatorname{cr}(A)}{\sum} Z(p)+\operatorname{dim} \prod_{\operatorname{fr}(G)} \underset{r(d A)}{\sum} Z(p)
$$


and for $n=0,1,2, \ldots$

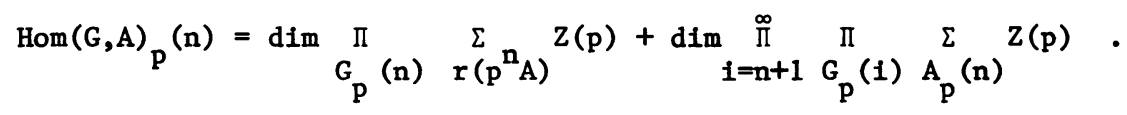

6. CONCLUSION.

Finally, it is easy to see that Theorem 5.5 allows us to calculate the Ulm invariants and the torsion free numbers for $\operatorname{Hom}(X, Y)$, where $X$ and $Y$ are arbitrary torsion groups.

Let $t_{p} X$ denote the $p$-primary component of $X$. Then $\operatorname{Hom}(X, Y) \cong \prod_{q} \operatorname{Hom}\left(t_{q} X, t_{q} Y\right)$, where $q$ ranges over the primes. Thus for each $n, \operatorname{Hom}(X, Y)_{p}(n)=\operatorname{Hom}\left(t_{p} X, t_{p} Y\right)_{p}(n)$. Also, since $\underset{q}{t \operatorname{HHom}}\left(t_{q} X, t_{q} Y\right)=\sum_{q} t \operatorname{Hom}\left(t_{q} X, t_{q} Y\right)$, it follows that

$\pi \operatorname{Hom}\left(t_{q} X, t_{q} Y\right) /\left(p \pi \operatorname{Hom}\left(t_{q} X, t_{q} Y\right)+t \pi \operatorname{Hom}\left(t_{q} X, t_{q} Y\right)\right) \cong$ $\operatorname{Hom}\left(t_{p} X, t_{p} Y\right) /\left(p H o m\left(t_{p} X, t_{p} Y\right)+t \operatorname{Hom}\left(t_{p} X, t_{p} Y\right)\right)$.

We have the following theorem.

THEOREM 6.1. If $X$ and $Y$ are torsion groups, then $\operatorname{Hom}(X, Y)$ is algebraically compact. Complete invariants for $\operatorname{Hom}(\mathrm{X}, \mathrm{Y})$ are given by

$\operatorname{Hom}(\mathrm{X}, \mathrm{Y})_{0}(\mathrm{p})=\operatorname{Hom}\left(\mathrm{t}_{\mathrm{p}} \mathrm{X}, \mathrm{t}_{\mathrm{p}} \mathrm{Y}\right)_{0}(\mathrm{p})$ and

Hom $(X, Y)_{p}(n)=\operatorname{Hom}\left(t_{p} X, T_{p} Y\right)_{p}(n)$, where $p$ ranges over the primes and $n$ ranges over the non-negative integers.

Hence the invariants calculated in Theorem 5.5 characterize Hom $(X, Y)$.

\section{REFERENCES}

1. Fuchs, L., Infinite Abelian Groups, Vol. 1, Academic Press, New York (1970).

2. Harrison, D. K., Infinite Abelian Groups and Homological Methods, Ann. of Math. 69 (1959) 336-391.

3. Legg, M. W. and E. A. Walker, An Algebraic Treatment of Algebraically Compact Groups, Rocky Mountain J. Math. $\underline{5}$ (1975) 291-299.

4. Maranda, J. M., On Pure Subgroups of Abelian Groups, Arch. Math. 11 (1960) 1-13. 
5. Pierce, R. S., Homomorphisms of Primary Abelian Groups, Topics in Abelian Groups, ed. by J. M. Irwin and E. A. Walker, Scott Foresman, Chicago (1963) 215-310.

6. Szele, T., On the Basic Subgroups of Abelian P-groups, Acta. Math. Acad. Sc1. Hung., 5 (1954) 129-140. 


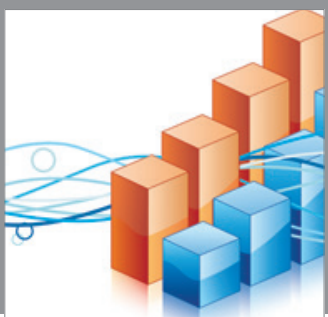

Advances in

Operations Research

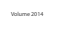

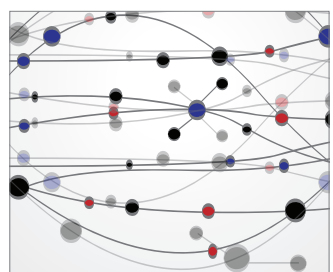

\section{The Scientific} World Journal
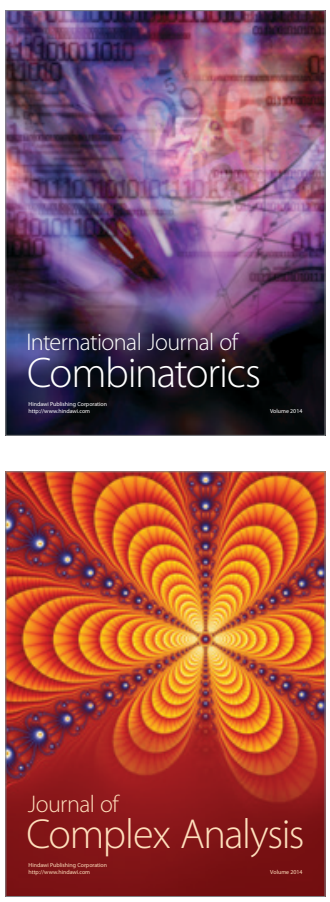

International Journal of

Mathematics and

Mathematical

Sciences
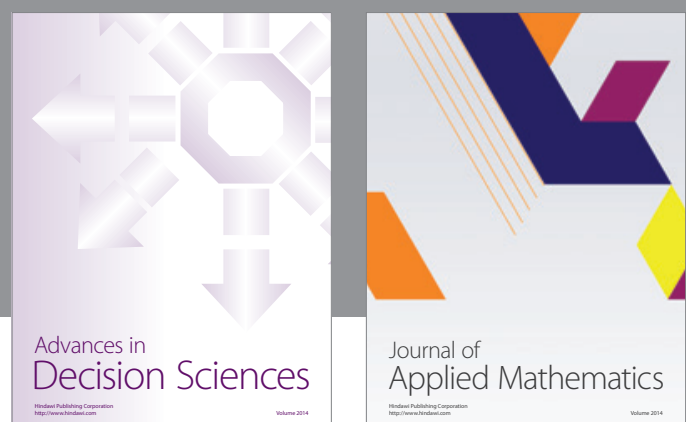

Journal of

Applied Mathematics
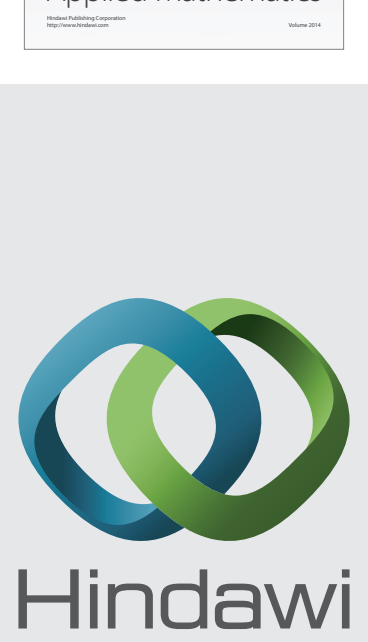

Submit your manuscripts at http://www.hindawi.com
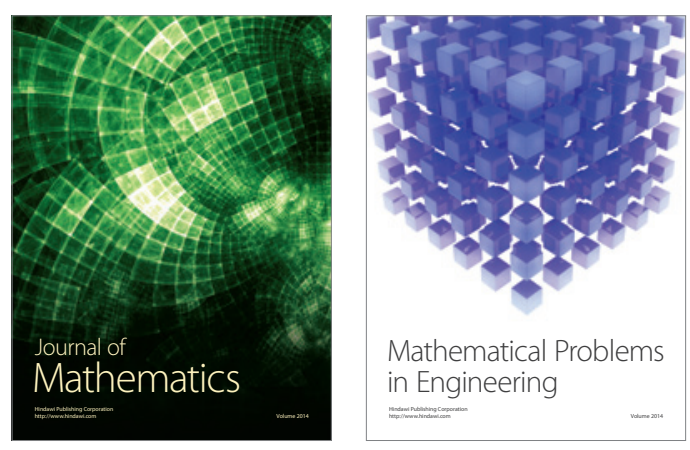

Mathematical Problems in Engineering
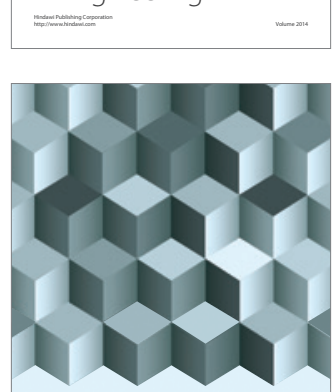

Journal of

Function Spaces
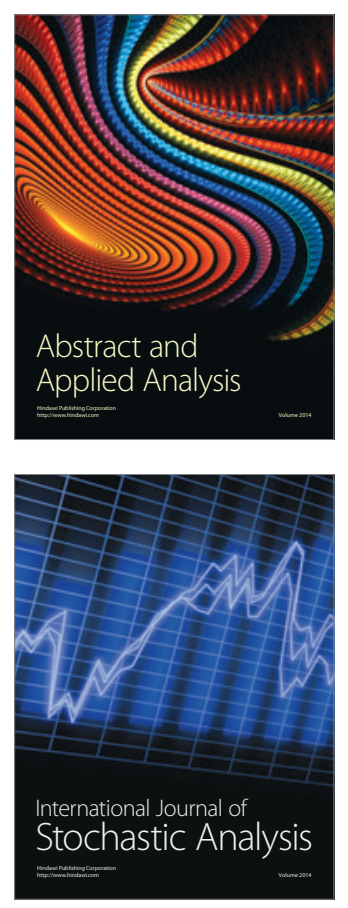

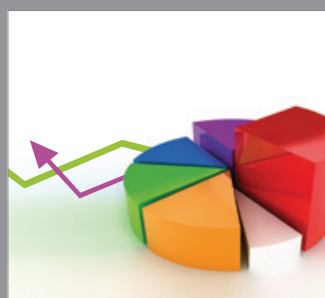

ournal of

Probability and Statistics

Promensencen
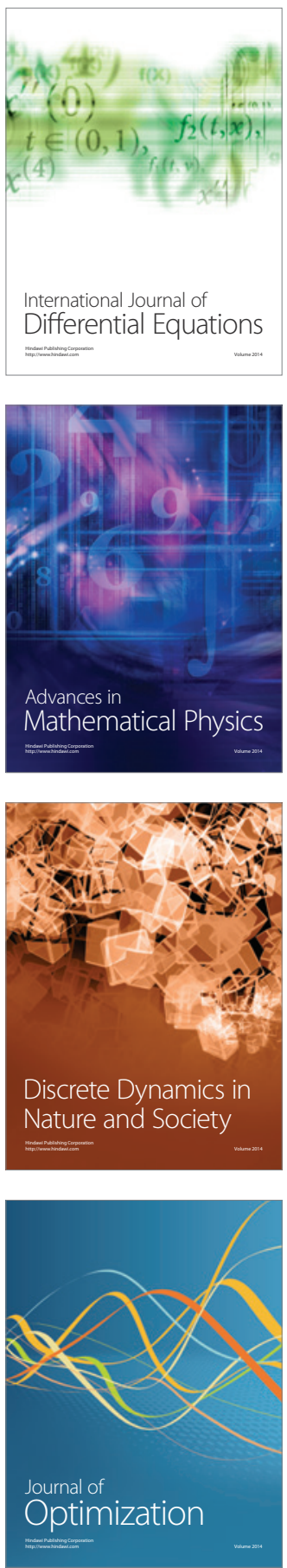